\title{
STABILITY OF THE CONTROLLED SYNCHRONIZATION MANIFOLD IN A RING OF MUTUALLY COUPLED CHAOTIC SYSTEMS
}

\author{
R. YAMAPI* \\ Department of Physics, Faculty of Science, \\ University of Douala, PO Box 24157 Douala, Cameroon \\ ryamapi@yahoo.fr \\ M. A. AZIZ-ALAOUI \\ Applied Mathematics Laboratory, University of Le Havre, \\ 25 rue ph. Lebon, B.P. 540, Le Havre, Cedex, France \\ aziz.alaoui@univ-lehavre.fr
}

Received October 22, 2007; Revised November 14, 2007

\begin{abstract}
The active control of the unstable synchronization manifold in a shift-invariant ring of $N$ mutually coupled chaotic oscillators is investigated. After deriving the bifurcation structures and chaotic states in the single oscillator, we find the regime of coupling parameters leading to stable and unstable synchronization phenomena in the ring, using the Master stability function approach with the transverse Lyapunov exponents. The active control technique is applied on the mutually coupled chaotic systems to suppress unstable synchronization states. We derive the range of control gain parameters which leads to a successful control and the stability of the control design. The effects of the amplitude of the parametric perturbations on the stability boundaries of the controlled unstable synchronization process are also studied.
\end{abstract}

Keywords: Synchronization; master stability function; Lyapunov exponents.

\section{Introduction}

Synchronization of mutually coupled nonlinear oscillators in the regular and chaotic states has been investigated for a long time by many researchers. This is due to the fact that synchronization is frequent in nature and can explain many phenomena in biology, chemistry, physics with various applications in engineering and communication [Pecorra et al., 1997; Nakata et al., 1998; Miyakawa \& Yamada, 2001; Strogatz \& Stewat, 1993; Kuramoto, 1984; Reimann et al., 1999]. In this spirit, the emergence of collective and synchronized dynamics in large networks of coupled units has also been investigated since the beginning of the nineties in different contexts and in a variety of fields, ranging from biology and ecology [Vannucchi \& Boccaletti, 2004; Hansel \& Sompolinsky, 1992; Pasemann, 1999], to semiconductor lasers [Winful \& Rahman, 1990; Li \& Erneux, 1993, 1994; Otsuka et al., 2000], to electronic circuits [Jankowski et al., 1995; Filatrella et al., 2001]. In particular, synchronization of mutually coupled systems is one of the most intensively investigated topics in nonlinear science [Heagy et al., 1994, 1995; Chen \& Zhou, 2006].

A crucial issue in the study of synchronization of mutually coupled systems is the assessment of

*Author for correspondence 
the stability of the synchronization process. As most of the practical implementations of such a process require to have stable synchronization regimes for all values of the coupling parameters, it is important to introduce strategies to suppress unstable synchronization. One possibility that has recently been proposed consists in coupling each unit to an external control force with the aim of stabilizing the synchronization manifold [Nana Nbendjo \& Yamapi, 2007].

We consider in this Letter the stability of the controlled synchronization manifold in a ring of mutually coupled chaotic systems. Our aim is to derive the range of control gain parameters which leads to successful control and the stability of the control design. After the onset of chaos states in the uncoupled driven oscillator, we begin by the derivation of different dynamical states which appear in the mutually coupled chaotic systems without control. For this aim, we use the Master stability function approach to derive the variation of the transverse Lyapunov exponents versus the coupling coefficient. The parametric active control technique is then applied to the mutually coupled systems to suppress undesired behavior such as unstable synchronization or to find the de-synchronization phenomena. The effects of the control or gain parameters in the control process and the amplitude of the parametric perturbations on the stable control domain are derived, and the results are shown in the stability diagram.

The Letter is organized as follows. In the next section we describe a ring of $N$ mutually coupled self-sustained electrical systems and derive the equations of motion. After presenting various bifurcation structures in the single oscillator in Sec. 3, the theory for the stability of the synchronous solution is given in Sec. 4 (master stability function approach). Attention is focused on the numerics of the stability analysis of the synchronization process of a shift-invariant ring of mutually coupled chaotic systems, and the types of behavior which appear on the shift-invariant ring. In Sec. 5, after presenting the model under the parametric active control, we analyze the stability of the control process using the Master stability function approach. The effects of the control parameters and the parametric perturbations on the synchronization manifold are derived. The paper ends with a short concluding section.

\section{Description of Mutually Coupled Systems}

The electrical model shown in Fig. 1 is a shiftinvariant ring of $N$ mutually coupling identical self-excited electrical systems. Each self-excited system (see Fig. 2) consists of a capacitor $C$, an inductor $L$ and a nonlinear resistor NLR, all connected in series. Two types of nonlinear components are considered: the voltage of the condenser is a nonlinear function of the instantaneous electrical charge $q_{\nu}$, which for the $\nu$ th unit is expressed as

$$
V_{c}^{\nu}=\frac{1}{C_{0}} q_{\nu}+a_{3} q_{\nu}^{3}
$$

where $C_{0}$ is the linear value of $C$ and $a_{3}$ is a nonlinear coefficient depending on the type of the capacitor in use. This is typical of nonlinear reactance components such as varactor diodes widely used in many areas of electrical engineering to design for instance parametric amplifiers, up-converters, mixers, low-power microwave oscillators, etc. [Oksasoglou \& Vavrim, 1994]. The current-voltage characteristics of a resistor for the $\nu$ th unit is also defined as

$$
V_{R_{0}}^{\nu}=R_{0} i_{0}\left[-\left(\frac{i_{\nu}}{i_{0}}\right)+\left(\frac{i_{\nu}}{i_{0}}\right)^{3}\right],
$$

where $R_{0}$ and $i_{0}$ are respectively, the normalization resistance and current, $i_{\nu}$ the value of current corresponding to the limit resistor voltage. In this case, the model has the property to exhibit selfexcited oscillations. This is due to the fact that the model incorporates through its nonlinear resistance a dissipative mechanism to damp oscillations that grow too large, and is also a source of energy to pump up those that become too small. The presence of such nonlinear resistors in each electrical system confers to the mechanism of self-sustained oscillations. The coupling between the $N$ identical self-sustained electrical models is realized through a capacitor $C_{m}$ (high-pass coupling). When the $N$ self-sustained electrical systems are interconnected as in Fig. 1, the Kirchhoff law of the voltage and current enables to find that the $k$ th self-sustained electrical system is described by the following equations

$$
V_{k}-V_{k+1}=V_{\text {self }},
$$

and

$$
I_{k-1}-I_{k}=I_{\text {self }}=C_{m} \frac{d V_{k}}{d \tau_{1}}
$$




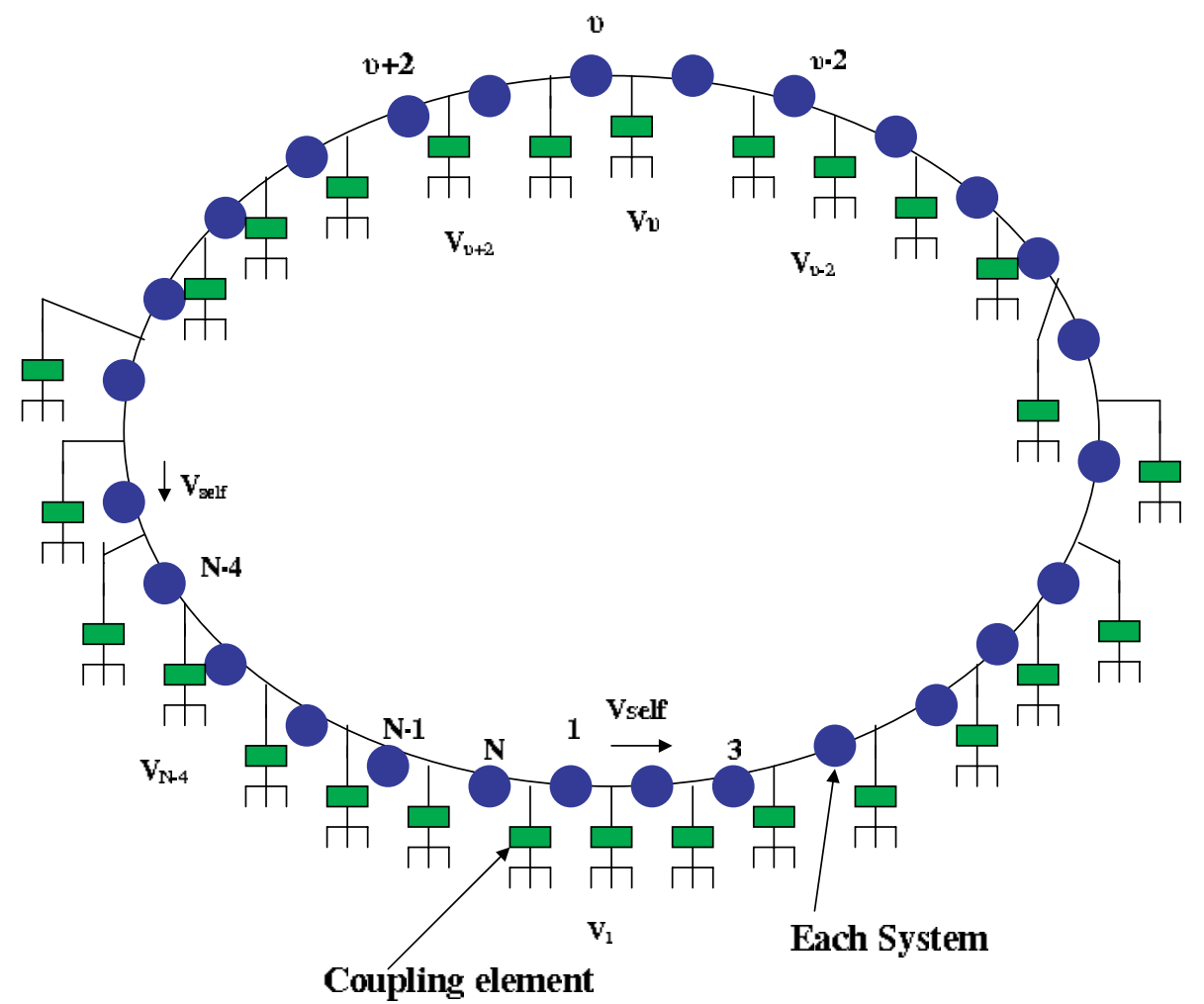

Fig. 1. A ring of mutually coupled self-sustained electrical systems.
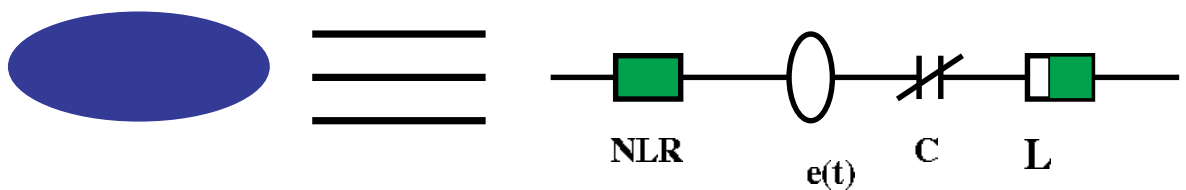

(a)

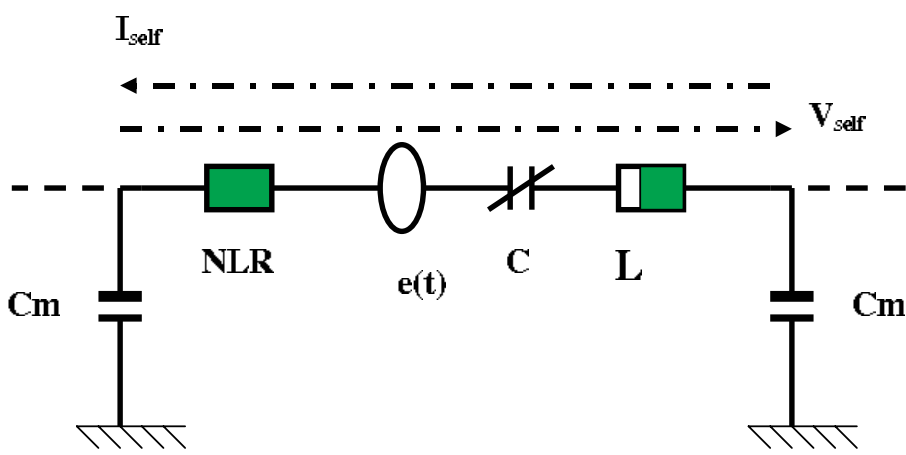

(b)

Fig. 2. (a) Schema of each electrical unit; (b) Alternative schema of a unit including the capacitors to ground at each end, which makes the unit forced self-exciting. 
which leads to

$$
V_{k}=\frac{1}{C_{m}}\left(q_{k-1}-q_{k}\right)
$$

Substituting Eq. (4) into Eq. (5) yields

$$
V_{\text {self }}=\frac{1}{C_{m}}\left(q_{k-1}-q_{k}\right)-\frac{1}{C_{m}}\left(q_{k}-q_{k+1}\right) .
$$

It is found that the ring of mutually coupled selfsustained electrical oscillators is described as

$$
\begin{gathered}
L \frac{d^{2} q_{\nu}}{d \tau_{1}^{2}}-R_{0}\left[1-\frac{1}{i_{0}^{2}}\left(\frac{d q_{\nu}}{d \tau_{1}}\right)^{2}\right] \frac{d q_{\nu}}{d \tau_{1}}+\frac{q_{\nu}}{C_{0}}+a_{3} q_{\nu}^{3} \\
=\nu_{0} \cos \Omega \tau_{1}+\frac{1}{C_{m}}\left(q_{\nu+1}-2 q_{\nu}+q_{\nu-1}\right), \\
\nu=1,2, \ldots, N
\end{gathered}
$$

with the following dimensionless variables

$$
x_{\nu}=\frac{q_{\nu}}{q_{0}}, \quad t=\omega_{e} \tau_{1},
$$

and

$$
\begin{gathered}
\alpha_{0}=\frac{q_{0}^{2} w_{e}^{2}}{i_{0}^{2}}, \quad \omega_{e}^{2}=\frac{1}{L C_{0}}, \quad \mu=\frac{R_{0}}{L \omega_{e}}, \quad \beta=\frac{a_{3} q_{0}^{2}}{L \omega_{e}^{2}}, \\
K=\frac{C_{0}}{C_{m}}, \quad E_{0}=\frac{v_{0}}{L q_{0} \omega_{e}^{2}}, \quad \omega=\frac{\Omega}{\omega_{e}} .
\end{gathered}
$$

Equation (7) yields the following set of coupled nondimensional differential equations

$$
\begin{array}{r}
\ddot{x}_{\nu}-\mu\left(1-\alpha_{0} \dot{x}_{\nu}^{2}\right) \dot{x}_{\nu}+x_{\nu}+\beta x_{\nu}^{3} \\
=E_{0} \cos w t+K\left(x_{\nu+1}-2 x_{\nu}+x_{\nu-1}\right) \\
\nu=1,2, \ldots, N
\end{array}
$$

where $x_{\nu}$ describes the components of the $\nu$ th self-sustained electrical system. $K$ is the coupling parameter, $E_{0}$ and $w$ are the amplitude and frequency of the external excitation. We fix $\alpha_{0}=1$, and introduce $y_{\nu}=\dot{x}_{\nu}$ to obtain

$$
\begin{aligned}
\dot{x}_{\nu}= & y_{\nu}, \\
\dot{y}_{\nu}= & \mu\left(1-y_{\nu}^{2}\right) y_{\nu}-x_{\nu}-\beta x_{\nu}^{3}+E_{0} \cos w t \\
& +K\left(x_{\nu+1}-2 x_{\nu}+x_{\nu-1}\right) \\
& \quad \nu=1,2, \ldots, N .
\end{aligned}
$$

Equations (9) are described by a set of $N$ mutually coupled identical Rayleigh-Duffing equations.
Thus, we have a shift-invariant ring of $N$ mutually coupled self-sustained electrical systems.

The great interest devoted to the model shown in Fig. 1 and described by Eqs. (8) is due to the fact that this model can be used to describe several phenomena and have applications in many areas. For instance, in electrical engineering, this model is used for a parallel operating system of microwave oscillators [Fukui \& Nagi, 1980, 1986]. A computer study of the mutual synchronization of a large number of these oscillators can be used to model intestinal signal [Robertson-Dunn \& Linkens, 1974] or colorectal myoelectrical activity in humans [Linkens et al., 1976].

\section{Bifurcation Structures and Chaos}

We find is this section various bifurcation structures which appear in the single or uncoupled RayleighDuffing system before the onset of chaos. For this purpose, the periodic stroboscopic bifurcation diagrams of the coordinate $x$ is used to map the transitions (the stroboscopic time period is $T=$ $2 \pi / w)$. We have found that chaos appears in the model for the physical parameters: $E_{0}=3.5, w=$ $1, \mu=\beta=5$. Figures 3-5 show a representative bifurcation diagram and the variation of the corresponding Lyapunov exponent versus some particular coefficient parameters. We have done the numerical calculations very accurately in double precision for different initial conditions and for different variations of parameters, and verified our results. In order to obtain reliable numerical results, the step size has been chosen to be equal to $10^{-4}$, and the first $10^{7}$ steps are discarded to avoid the transient regime. These curves are obtained by solving numerically, Eq. (8) with $K=0$ and the corresponding variational equations, the Lyapunov exponent being defined by

$$
\text { Lya }=\lim _{t \rightarrow+\infty} \frac{\ln \left(\sqrt{d x^{2}+d \dot{x}^{2}}\right)}{t}
$$

where $d x$ and $d \dot{x}$ are respectively the variations of $x$ and $\dot{x}$. That is a measure of the rate of divergence between initially closed trajectories in the twodimensional phase space. As it appears, different types of bifurcations take place before the onset of chaos. In Fig. 3, we find that as $E_{0}$ increases from zero, the amplitude of the quasi-periodic oscillations increases until $E_{0}=2.41$ where the quasi-periodic 


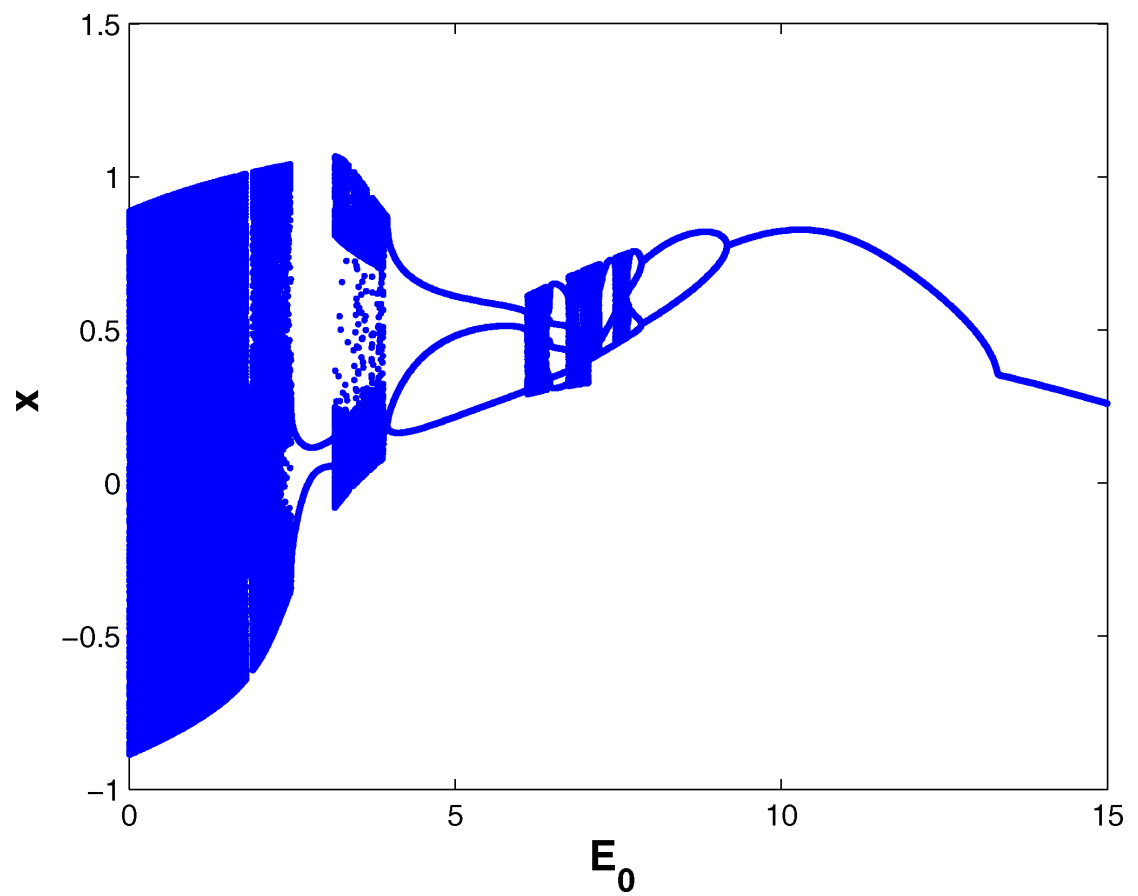

(a)

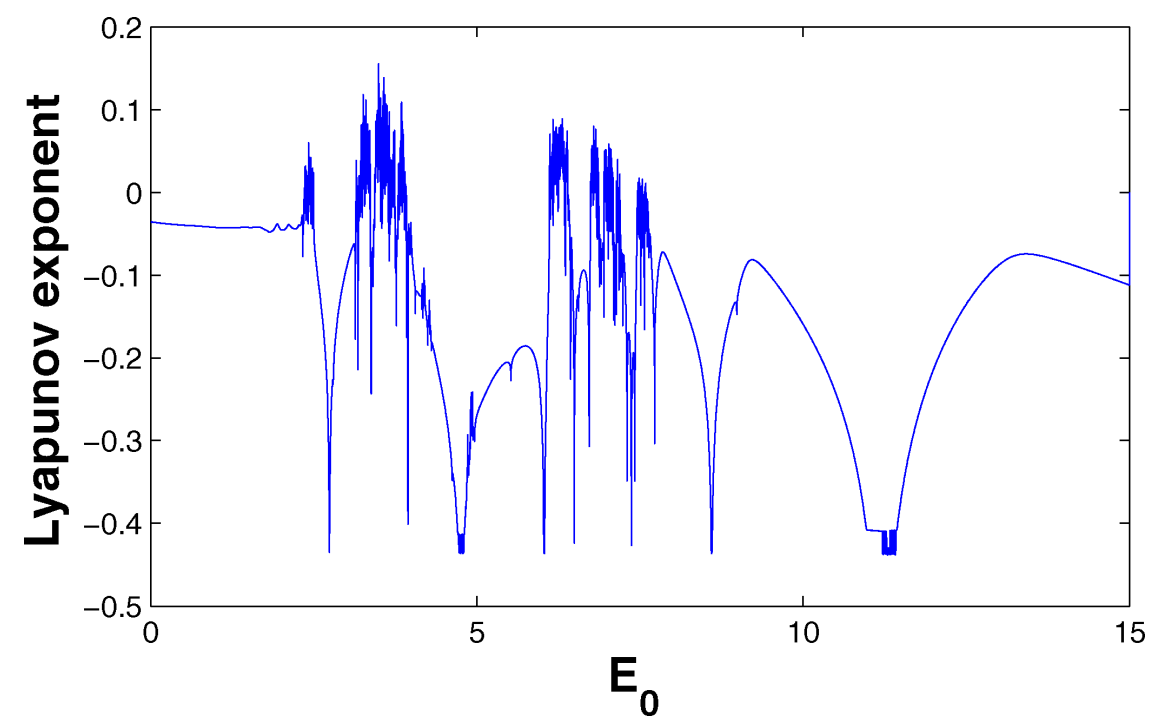

(b)

Fig. 3. (a) Bifurcation diagram and (b) variation of the Lyapunov exponent versus $E_{0}$ with $w=1$ and $\mu=\beta=5$.

behavior bifurcates into a small window of chaos. Then after the value $E_{0}=2.45$, a periodic transition appears and the system passes into $2 \mathrm{~T}$-periodic state. As $E_{0}$ increases further, the 2T-period orbit bifurcates to an other chaotic states and the perioddoubling cascade continues leading to a chaotic window and periodic orbit. In summary, it appears that the chaotic states are found in the region of $E_{0}$ defined as $E_{0} \in[2.41 ; 2.45] \cup[3.18 ; 3.36] \cup$ $[3.44 ; 3.65] \cup[3.71 ; 3.73] \cup[3.83 ; 3.9] \cup[6.14 ; 6.39] \cup$ $[6.74] \cup[6.86] \cup[6.95 ; 7.07] \cup[7.14 ; 7.16]$. Figures 4 and 5 also show the bifurcation structures and 


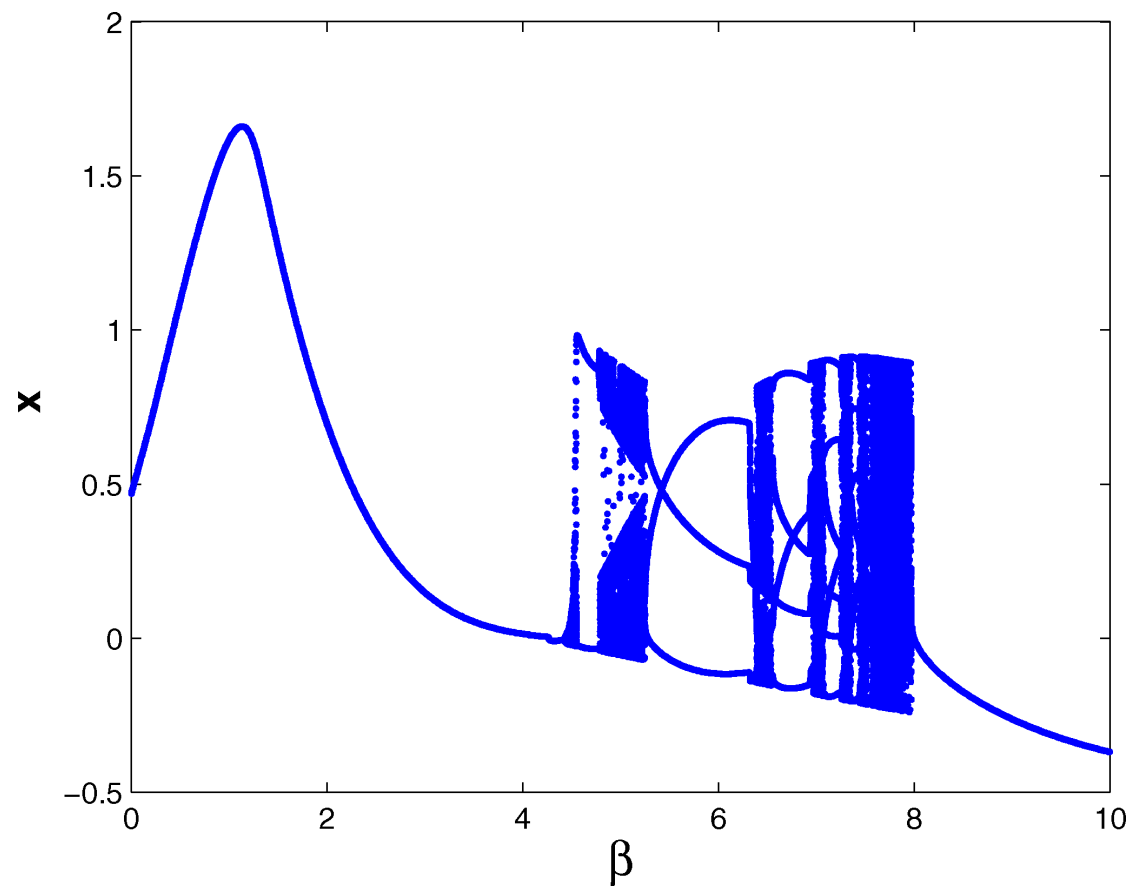

(a)

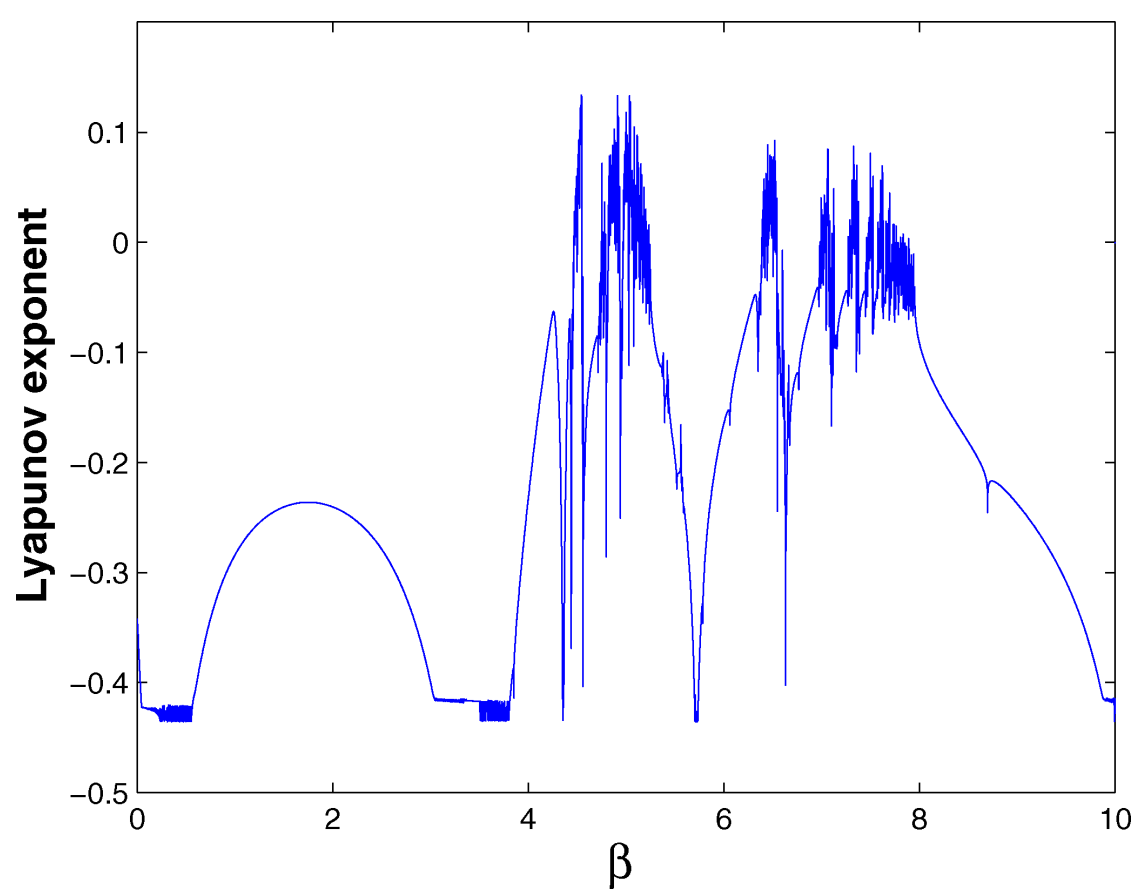

(b)

Fig. 4. (a) Bifurcation diagram and (b) variation of the Lyapunov exponent versus $\beta$ with $w=1$ and $\alpha=5, E_{0}=3.5$.

the variation of the Lyapunov exponent versus $\beta$ (for fixed $\mu=5, E_{0}=3.5$ ) and $\mu$ (for fixed $\beta=5, E_{0}=3.5$ ), respectively. For example, when $\mu=5$, the chaotic states appear for $\beta \in$ $[4.47 ; 4.54] \cup[4.74 ; 4.78] \cup[4.82 ; 4.92] \cup[4.97 ; 5.18] \cup$
$[6.39 ; 6.54] \cup[7.02 ; 7.07] \cup[7.31 ; 7.35] \ldots$, while for $\beta=5$, chaos appears for $\mu \in[3.18 ; 3.25] \cup$ $[4.67 ; 4.72] \cup[4.81 ; 5.05] \cup[5.12 ; 5.23] \cup[6.05 ; 6.12] \cup$ $[6.16 ; 6.24] \cup[6.32 ; 6.45] \cup[6.5 ; 6.55] \cup[6.94 ; 7.1] \cup$ $[7.16 ; 7.45] \cup[7.84 ; 8.49]$. 


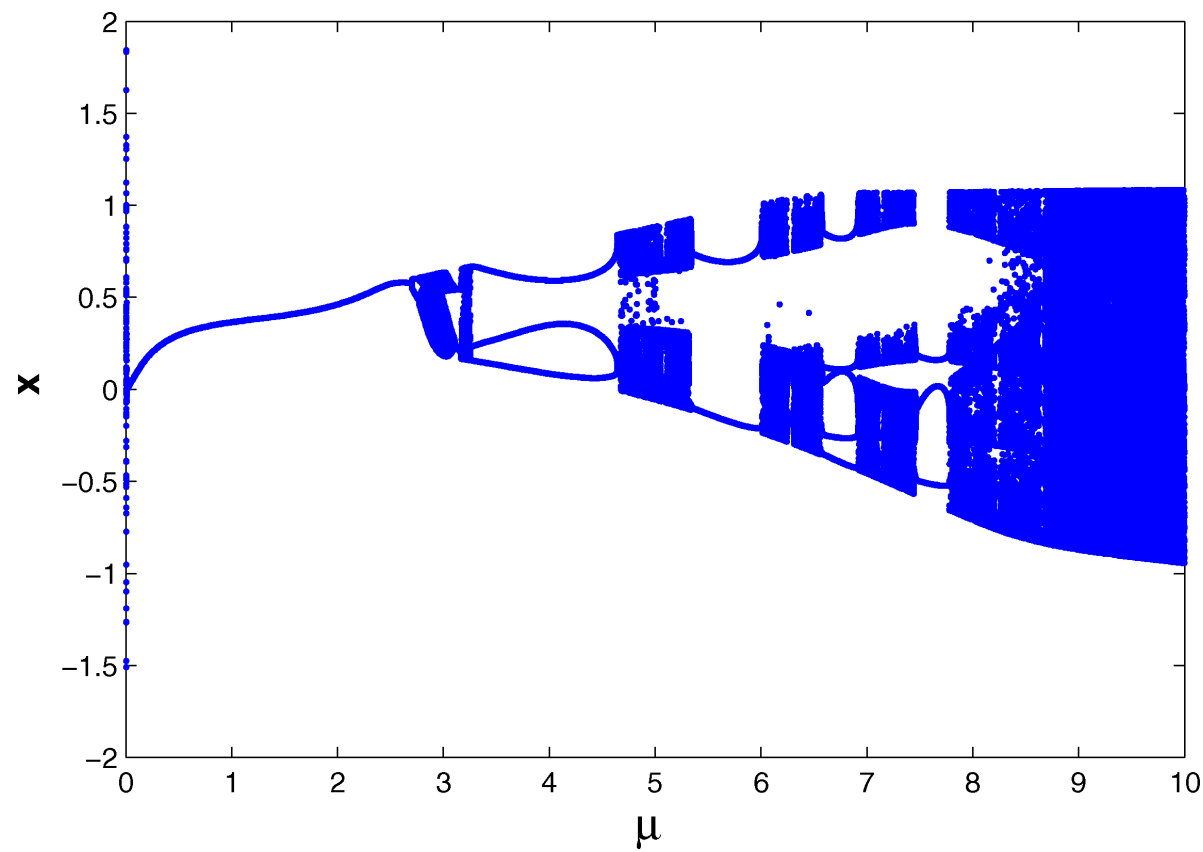

(a)

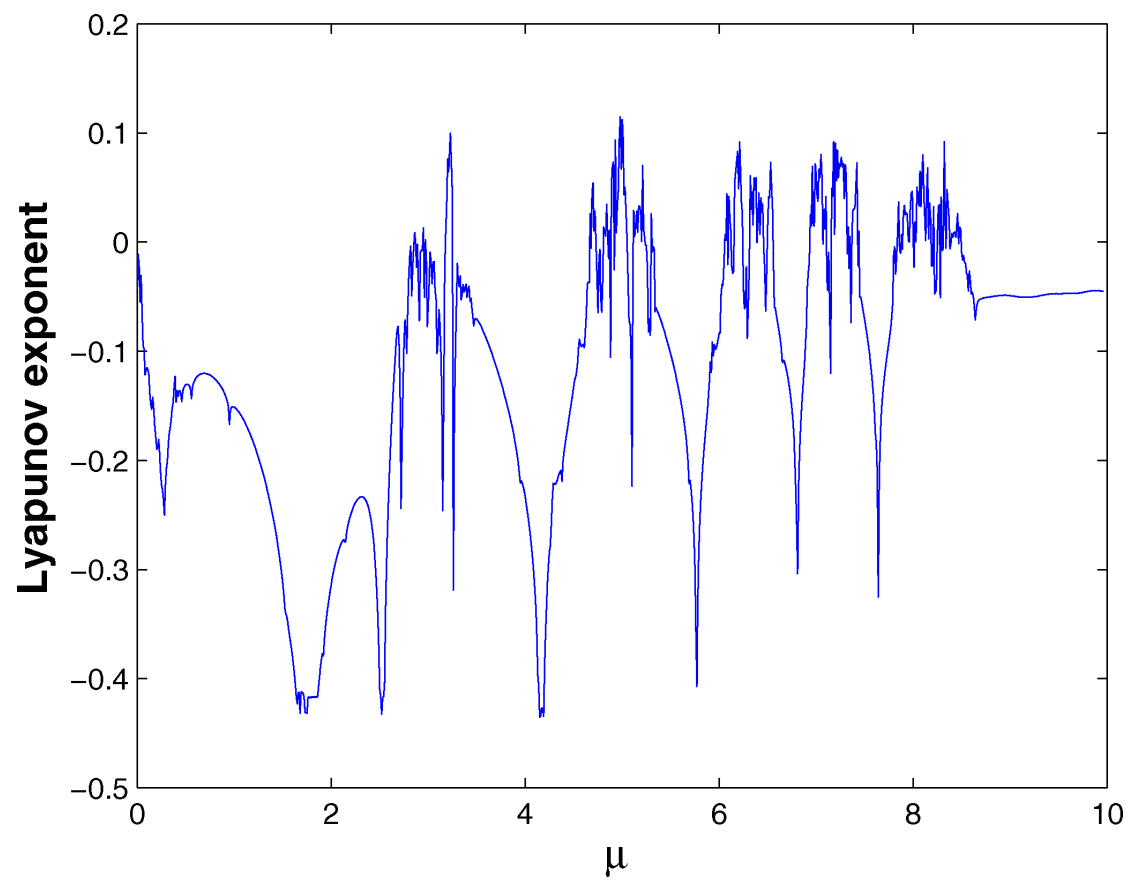

(b)

Fig. 5. (a) Bifurcation diagram and (b) variation of the Lyapunov exponent versus $\mu$ with $w=1$ and $\beta=5, E_{0}=3.5$.

\section{Synchronization Analysis}

\subsection{The Master stability function approach}

Various dynamical states which appear in the shiftinvariant ring depending on the coupling strength $K$ and the number $N$ of self-sustained electrical systems are identified here. The model is physically interesting so long as the dynamical states are stable. As we are interested in bifurcations from synchronous chaotic states; these states reside on a synchronization manifold defined by $\mathcal{M}=\left\{x_{1}=\right.$ 
$\left.x_{2}=\cdots=x_{N} ; y_{1}=y_{2}=\cdots=y_{N}\right\}$, which has the dimension two of the single chaotic self-sustained electrical system. The central goal of this investigation is the ability to predict when the synchronized state defined by $x_{k}=x_{s}(t) ; y_{k}=y_{s}(t)$, is stable $\forall k$, representing the stable synchronization manifold $\mathcal{M}$, whose stability will be the object of the study. In what follows, we present the stability analysis of the synchronized state of a shift-invariant ring of mutually coupled Rayleigh-Duffing systems. The master stability approach [Boccaletti et al., 2006; Pecora \& Carroll, 1998] will be used to find the stable chaos synchronization in the ring. For this purpose, let $\mathbf{X}^{i}$ be the two-dimensional vector of the dynamical variables of the $i$ th unit, $\mathbf{H}: R^{2} \rightarrow R^{2}$ an arbitrary function of each unit variables used in the coupling. Thus, the dynamics of the $i$ th unit are rewritten as a function of the $2 \times N$ column vector state $\mathbf{X}^{i}$ as

$\dot{\mathbf{X}}^{i}=\mathbf{F}\left(\mathbf{X}^{i}\right)+K \sum_{j}^{N} G_{i j} \mathbf{H}\left(\mathbf{X}^{j}\right), \quad i=1,2, \ldots, N$,

where $\mathbf{X}^{i}=\left[x_{i}, y_{i}\right]^{T}, \mathbf{F}\left(\mathbf{X}^{i}\right)=\left[y_{i}, \mu\left(1-y_{i}^{2}\right) y_{i}-x_{i}-\right.$ $\left.\beta x_{i}^{3}\right]^{T}$, and the function $\mathbf{H}$ is defined through the matrix

$$
\mathbf{E}=\left(\begin{array}{ll}
0 & 0 \\
1 & 0
\end{array}\right)
$$

by $\mathbf{H}\left(\mathbf{X}^{i}\right)=\mathbf{E} \mathbf{X}^{i} . G_{i j} \in R$ are $N \times N$ symmetry connectivity matrix $G$ in the shift-invariant ring of mutually chaotic coupled oscillators and defined by

$$
\mathbf{G}=\left(\begin{array}{rrrrr}
-2 & 1 & 0 & \ldots & 1 \\
1 & -2 & 1 & \ldots & 0 \\
0 & 1 & -2 & \ldots & \vdots \\
\vdots & \vdots & \vdots & \ddots & 1 \\
1 & 0 & \ldots & 1 & -2
\end{array}\right)
$$

and adheres to the zero row-sum condition: $\sum_{j=1}^{n} G_{i j}=0$ for all $i$. The synchronization manifold $\mathcal{M}$ is an invariant set, due to the zero rowsum condition of the coupling matrix $G$ and due to the fact that the function $\mathbf{H}(\mathbf{X})$ is the same for all shift-invariant ring units. Therefore, stability of the synchronous state reduces to satisfy the system's dynamical properties along directions in phase that are transverse to the synchronization manifold. Thus, the stability of the resulting dynamical states can be determined by letting $x_{\nu}=\delta x_{\nu}+x_{s}, y_{\nu}=\delta y_{\nu}+y_{s}$ and linearizing equations (11) around the state $\left(x_{s}, y_{s}\right)$. This leads to

$$
\delta \dot{\mathbf{X}}=\left[1_{N} \otimes J \mathbf{F}\left(\mathbf{X}_{s}\right)+K G \otimes J \mathbf{H}\left(\mathbf{X}_{s}\right)\right] \delta \mathbf{X},
$$

where $\otimes$ stands for the direct product between matrices, $J$ denotes the Jacobian operator and the $2 \times N$ column vectors $\delta \mathbf{X}^{i}=\left(\delta x_{i}, \delta y_{i}\right)$ is the deviation of the $i$ th vector state from the synchronization manifold. Note that $\mathbf{H}$ is just a matrix $\mathbf{E}$ and $J \mathbf{H}=E$.

A necessary condition for stability of the synchronization manifold [Boccaletti et al., 2006; Pecora \& Carroll, 1998] is that the set of $(N-1) * 2$ Lyapunov exponents that corresponds to phase space directions transverse to the 2-dimensional hyperplane $\mathbf{X}^{i}=\mathbf{X}_{s}$ be entirely made of negative values. Equation (12) is used to calculate Lyapunov exponents. Since Eqs. (12) are high-dimensional, the synchronization problem is to reduce this highdimensional set of variational equations, governing the stability of the synchronous state, to a more manageable, low-dimensional set. We really want to consider only variations which are transverse to the synchronization manifold. We next show how to separate out those variations and simplify the problem. Let $\gamma_{i}$ and $v_{i}$ be the set of real eigenvalues and the associated orthonormal eigenvectors of the matrix $G$ respectively, such that $G v_{i}=\gamma_{i} v_{i}$ and $v_{j}^{T} \cdot v_{i}=\delta_{i j}$. The arbitrary state $\delta \mathbf{X}$ can be written as $\delta \mathbf{X}(t)=\sum_{i=1}^{N} v_{i} \otimes \xi_{i}(t)$ with $\xi_{i}(t)=$ $\left(\xi_{i, 1}(t), \xi_{i, 2}(t)\right)$. If one applies $v_{j}^{T}$ to the left side of each equations (12), one finally obtains the following set of $N$ variational equations

$$
\begin{array}{r}
\dot{\xi}_{k}=\left[J \mathbf{F}\left(\mathbf{X}_{s}\right)+K \gamma_{k} J \mathbf{H}\left(\mathbf{X}_{s}\right)\right] \xi_{k}, \\
k=0,1,2, \ldots, N-1,
\end{array}
$$

in the evolution kernel. We recall that $\gamma_{k}$ is the eigenvalue of $G$, and is given by $\gamma_{k}=-4 \sin ^{2}(\pi k / N)$ for the diffusive coupling configuration [Pecora \& Carroll, 1998]. One notes that each equation in (13) corresponds to a set of two conditional Lyapunov exponents $\lambda_{k}^{j}(j=1,2)$ along the eigenmode corresponding to the specific eigenvalue $\gamma_{k}$. For $k=0$, we have the variational equation for the synchronization manifold and its maximum Lyapunov exponent $\lambda_{\max }^{0}=0.191$ is that of the isolated chaotic dynamical unit. The remaining variations $\xi_{k}, k=1,2, \ldots, N-1$ are transverse to $\mathcal{M}$, and describe the system's response to small deviation from the synchronization manifold, and then control the stability of the synchronized state. Any 


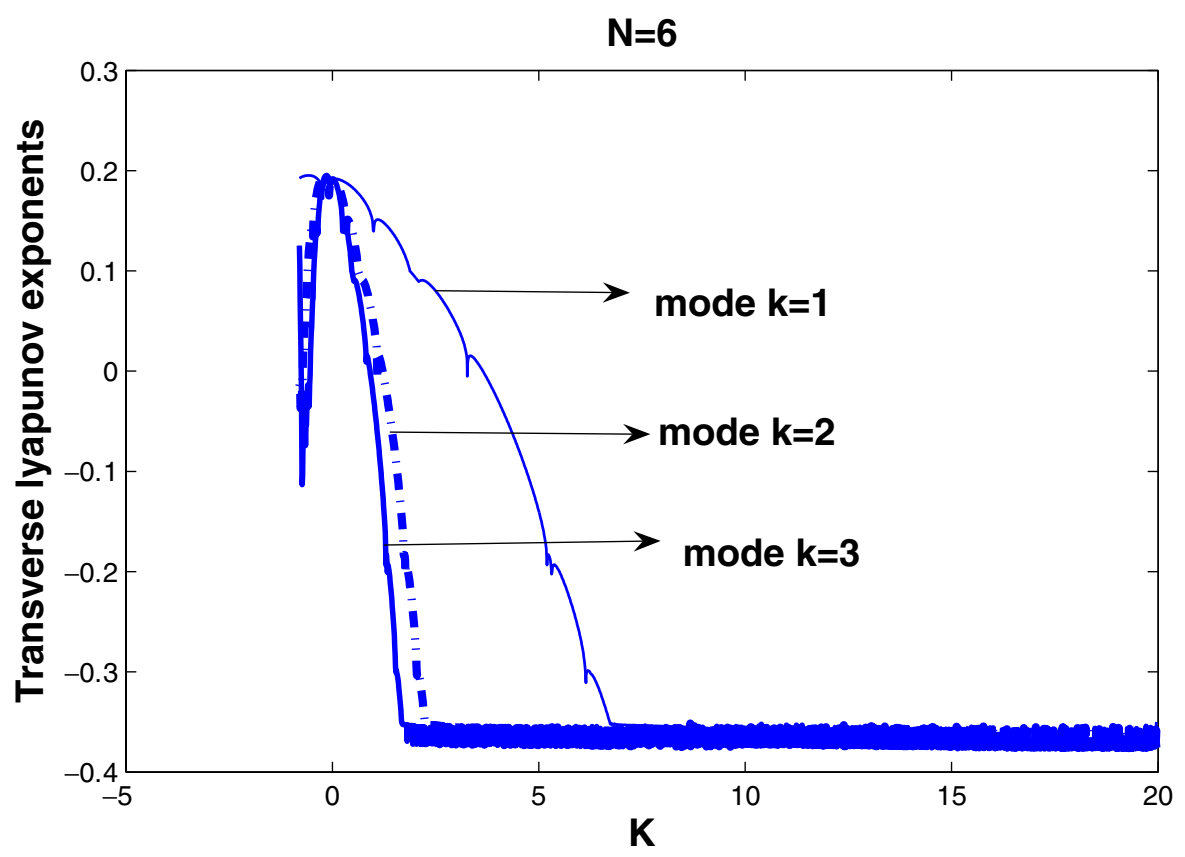

(a)

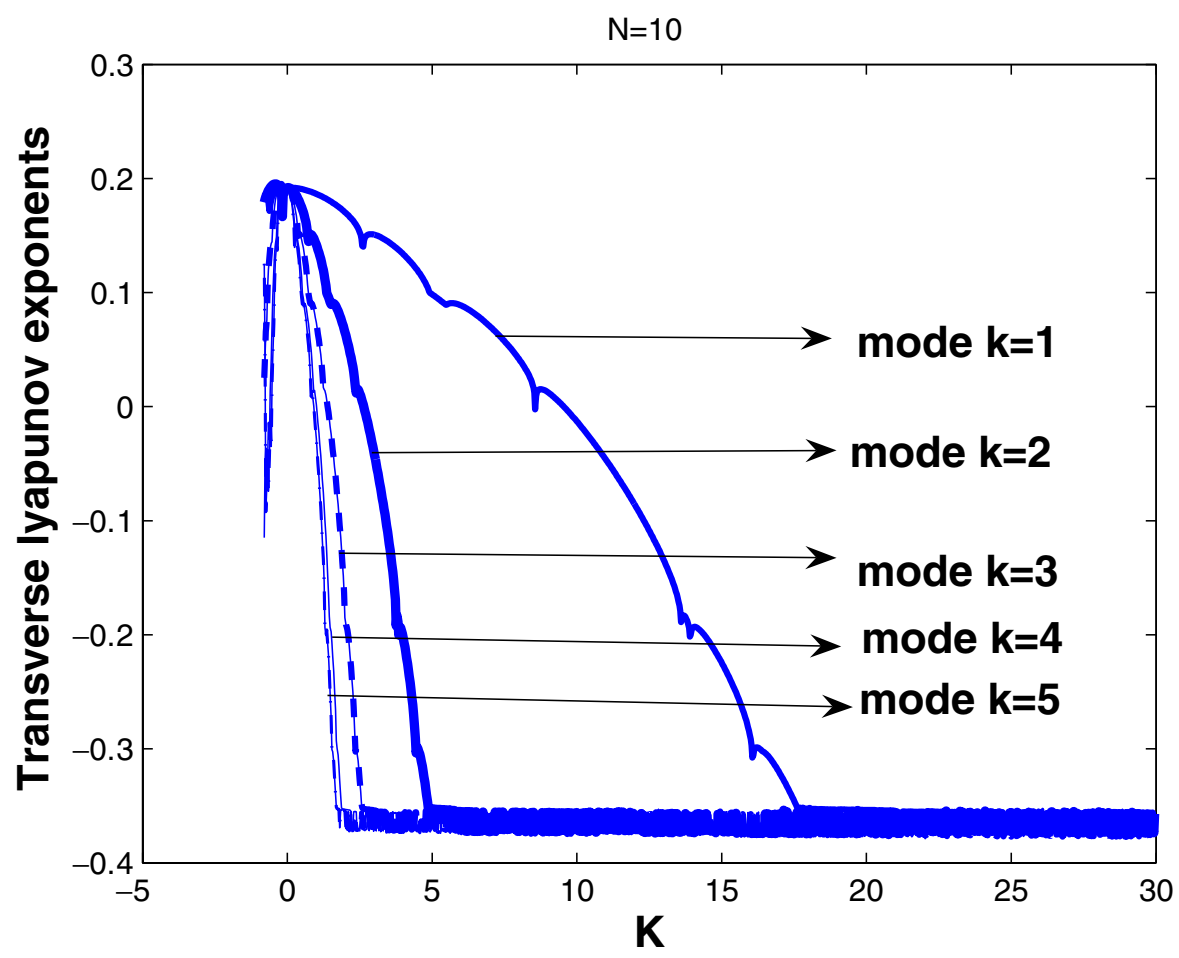

(b)

Fig. 6. Variations of the transverse Lyapunov exponents versus $K$ with $w=1$ and $\beta=\mu=5, E_{0}=3.5$. 
deviation from the synchronization manifold will be reflected in the growth of one or more of these variations. We note that the stability of the synchronized state is ensured if arbitrarily small transverse variations decay to zero. For $k \neq 0$, Eqs. (13) enables to calculate the maximum transverse Lyapunov exponent $\lambda_{k}^{\max }$ of each mode $k$ as the function of the coupling parameter $K$. We recall that the synchronization state is stable if all the maximum Lyapunov exponents $\lambda_{\max }^{k}$ of the transverse modes are negative.

\subsection{Numerical simulations}

Now we report in this subsection on numerical computation of the Lyapunov exponents and determine the domain of stable synchronization. When the coupling coefficient is turned off (i.e. $K$ is equal to zero), the chaotic driven self-sustained electrical systems are uncoupled. The corresponding maximum Lyapunov exponents are $\lambda_{0}^{\max }=0.191$ for the physical set of parameters used in the following analysis of the paper: $E_{0}=3.5 ; w=1 ; \mu=\beta=5$. For $K \neq 0$, and for a given value of $N$, the maximum transverse Lyapunov exponents will enable us to derive the range of the coupling parameter $K$ in which the transverse Fourier modes are stable, and then each of a group of systems of the ring works in a synchronizing manner.

The variation of the transverse Lyapunov exponents are derived and plotted on Fig. 6. As $K$ increases, two different dynamical states appear in the mutually coupled systems: The first is the unstable domain in which there is some positive Lyapunov exponent and hardly depends on the number $N$, no synchronization is observed, all the modes are on the transverse manifold where variations transverse to the synchronization manifold do not decay with the time since all the transverse Lyapunov exponents are positive i.e. $\lambda_{k}^{\max }>0$. In the second, the synchronization process is observed and all the transverse Lyapunov exponents are negative. In this case, all the modes move from the unstable domain to the stable one, the ring is synchronized and stable synchronization is observed. The results are shown in Table 1 where the boundaries of stable synchronization are presented as a function of the coupling coefficient $K$. It appears that the domains of stable synchronization reduce dramatically and there is no domain of stable synchronization for negative values of the coupling coefficient $K$.
Table 1. Dynamical states in the mutually coupled chaotic systems.

\begin{tabular}{cccc}
\hline Number $N$ & $\begin{array}{c}\text { Domains of } \\
K \text { for (SS) }\end{array}$ & Number $N$ & $\begin{array}{c}\text { Domains of } \\
K \text { for }(\text { SS })\end{array}$ \\
\hline 2 & {$[0.92 ;+\infty]$} & 11 & {$[11.49 ;+\infty[$} \\
3 & {$[1.22 ;+\infty]$} & 12 & {$[13.61 ;+\infty[$} \\
4 & {$[1.83 ;+\infty]$} & 13 & {$[15.92 ;+\infty[$} \\
5 & {$[2.64 ;+\infty]$} & 14 & {$[18.42 ;+\infty[$} \\
6 & {$[3.65 ;+\infty[$} & 15 & {$[21.1 ;+\infty[$} \\
7 & {$[4.85 ;+\infty[$} & 16 & {$[23.96 ;+\infty[$} \\
8 & {$[6.23 ;+\infty[$} & 17 & {$[27.01 ;+\infty[$} \\
9 & {$[7.8 ;+\infty[$} & 18 & {$[30.24 ;+\infty[$} \\
10 & {$[9.5 ;+\infty[$} & 19 & {$[33.66 ;+\infty[$} \\
& & 20 & {$[37.26 ;+\infty[$} \\
\hline
\end{tabular}

(SS): Stable synchronization.

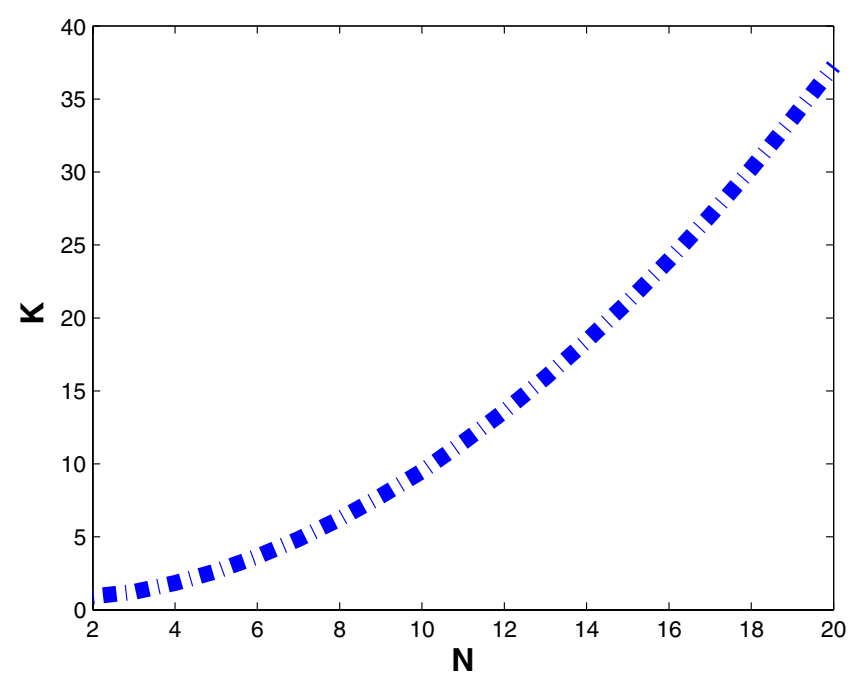

Fig. 7. Stability boundaries of the synchronization in the $(K, N)$ plan with $w=1$ and $\beta=5, E_{0}=3.5$.

Figure 7 is the stability diagram, that displays the two main dynamical states and the stability boundaries and we find that the number of units hardly affects the stability boundaries of the synchronization process in the ring.

\section{Active Control}

\subsection{Mathematical formulation}

The main outcome of the previous analysis is that the synchronization process is stable or unstable, depending upon the coupled coefficient $K$. It is then interesting to study how unstable synchronization can be controlled, and devise an active control strategy to suppress the undesired behavior. To illustrate 
that, we consider the following equations modeling the controlled system

$$
\begin{gathered}
\ddot{x}_{\nu}-\mu\left(1-\dot{x}_{\nu}^{2}\right) \dot{x}_{\nu}+x_{\nu}+\beta x_{\nu}^{3} \\
\quad+\lambda_{1}(1+q \cos 2 w t) \dot{u}_{\nu} \\
=E_{0} \cos w t+K\left(x_{\nu+1}-2 x_{\nu}+x_{\nu-1}\right) \\
\ddot{u}_{\nu}+\dot{u}_{\nu}+u_{\nu}-\lambda_{2}(1+q \cos 2 w t) \dot{x}_{\nu}=0 \\
\nu=1,2, \ldots, N
\end{gathered}
$$

where $u_{\nu}$ is the control force of the $\nu$ th selfsustained electrical system, $\lambda_{i}$ the control gain parameters, $q$ is the amplitude of the parametric coupling with $0 \leq q<1$. Practically, this type of control can be carried out in engineering by making use of a mechanical system, put together to the original self-sustained electrical system. The original self-sustained electrical system and the mechanical system are coupled to the electromagnetic force to form a self-sustained electromechanical system such as those presented in [Yamapi et al., 2003; Yamapi \& Bowong, 2006]. The origin of the parametric coupling is due to the fact that some parameters of the electromechanical device can vary with time because of the functioning constraints. This is particulary the case for the parameters of the electromagnetic coupling e.g. time variations of the magnetic field and the region of electromagnetic action. We assume that the time variation is periodic with frequency $2 w$.

Introducing $v_{k}=\dot{u}_{k}$, Eqs. (14) yield

$$
\begin{aligned}
\dot{x}_{\nu}= & y_{\nu} \\
\dot{y}_{\nu}= & \mu\left(1-y_{\nu}^{2}\right) y_{\nu}-x_{\nu}-\beta x_{\nu}^{3} \\
& -\lambda_{1}(1+q \cos 2 w t) v_{\nu}+E_{0} \cos w t \\
& +K\left(x_{\nu+1}-2 x_{\nu}+x_{\nu-1}\right) \\
\dot{u}_{\nu}= & v_{\nu} \\
\dot{v}_{\nu}= & -v_{\nu}-u_{\nu}+\lambda_{2}(1+q \cos 2 w t) y_{\nu} \\
& \quad \nu=1,2, \ldots, N,
\end{aligned}
$$

$q$ is the amplitude of the parametric perturbations on the gain coupled elements. We remind that we are interested in bifurcations from synchronous states; which reside on a synchronization manifold defined by $\mathcal{N}=\left\{x_{1}=x_{2}=\cdots=x_{N} ; y_{1}=\right.$ $y_{2}=\cdots=y_{N} ; u_{1}=u_{2}=\cdots=u_{N} ; v_{1}=$ $\left.v_{2}=\cdots=v_{N}\right\}$.

\subsection{Stability of the control process}

The Master stability approach [Boccaletti et al., 2006; Pecora \& Carroll, 1998] can be also used to find the appropriate range of the control gain parameters. For this purpose, let $\mathbf{Y}^{i}$ be the fourdimensional vector of the dynamical variables of the $i$ th unit. Equations (14) can be rewritten as

$\dot{\mathbf{Y}}^{i}=\mathbf{F}\left(t, \mathbf{Y}^{i}\right)+K \sum_{j}^{N} G_{i j} \mathbf{H}\left(\mathbf{Y}^{j}\right), \quad i=1,2, \ldots, N$,

where $\mathbf{Y}^{i}=\left[x_{i}, y_{i}, u_{i}, v_{i}\right]^{T}, \mathbf{F}\left(t, \mathbf{Y}^{i}\right)=\left[y_{i}, \mu(1-\right.$ $\left.y_{i}^{2}\right) y_{i}-x_{i}-\beta x_{i}^{3}-\lambda_{1}(1+q \cos 2 w t) v_{i}, v_{i},-v_{i}-$ $\left.u_{i}+\lambda_{2}(1+q \cos 2 w t) y_{i}\right]^{T}$. As in the above section, the stability of the control reduces to take care of the system's dynamical properties along directions in phase that are transverse to the synchronization manifold. Thus, the stability of the resulting dynamical states can be determined by letting $x_{\nu}=\delta x_{\nu}+x_{s}, y_{\nu}=\delta y_{\nu}+y_{s}, u_{\nu}=\delta u_{\nu}+u_{s}$, $v_{\nu}=\delta v_{\nu}+v_{s}$ and linearizing Eqs. (16) around the state $\left(x_{s}, y_{s}, u_{s}, v_{s}\right)$. This leads to

$$
\delta \dot{\mathbf{Y}}=\left[1_{N} \otimes J \mathbf{F}\left(t, \mathbf{Y}_{s}\right)+K G \otimes J \mathbf{H}\left(\mathbf{Y}_{s}\right)\right] \delta Y,
$$

where the $4 \times N$ column vector $\delta \mathbf{Y}^{i}=\left(\delta x_{i}, \delta y_{i}\right.$, $\left.\delta u_{i}, \delta v_{i}\right)$ is the deviation of the $i$ th vector state from the controlled synchronization manifold. We remind that $\mathbf{H}$ is also defined through the matrix $E$, and $J \mathbf{H}=E$ with

$$
E=\left(\begin{array}{llll}
0 & 0 & 0 & 0 \\
1 & 0 & 0 & 0 \\
0 & 0 & 0 & 0 \\
0 & 0 & 0 & 0
\end{array}\right)
$$

The arbitrary state $\delta \mathbf{Y}$ can be written as $\delta \mathbf{Y}(t)=$ $\sum_{i=1}^{N} v_{i} \otimes \eta_{i}(t)$ with $\eta_{i}(t)=\left(\eta_{i, 1}(t), \eta_{i, 2}(t), \eta_{i, 3}(t)\right.$, $\left.\eta_{i, 4}(t)\right)$. If one applies $v_{j}^{T}$ to the left-hand side of each equation (17), one finally obtains the following set of $N$ variational equations

$$
\begin{array}{r}
\dot{\eta}_{k}=\left[J \mathbf{F}\left(\mathbf{Y}_{s}\right)+K \gamma_{k} J \mathbf{H}\left(\mathbf{Y}_{s}\right)\right] \eta_{k}, \\
k=0,1,2, \ldots, N-1,
\end{array}
$$

As $\gamma_{k}$ is the eigenvalue of $G$, it is given as $\gamma_{k}=-4 \sin ^{2}(\pi k / N)$ for the diffusive coupling [Pecora \& Carroll, 1998], and each equation in (18) corresponds to a set of four conditional Lyapunov exponents $\lambda_{k}^{j}(j=1,2,3,4)$ along the eigenmode corresponding to the specific eigenvalue $\gamma_{k}$. For $\lambda_{i} \neq 0$, Eqs. (17) enables to calculate the maximum transverse Lyapunov exponent $\lambda_{k}^{\max }$ of each mode $k$ as the function of the coupling gain parameter $\lambda_{i}$. 


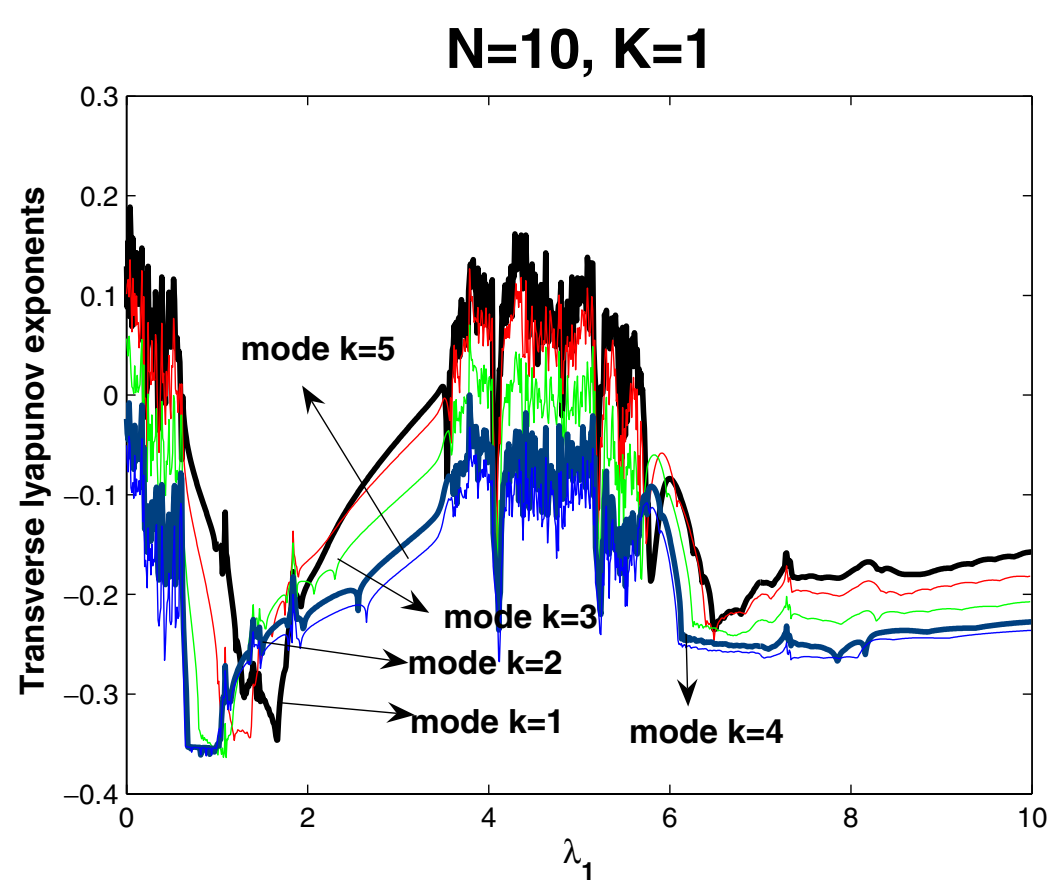

(a)

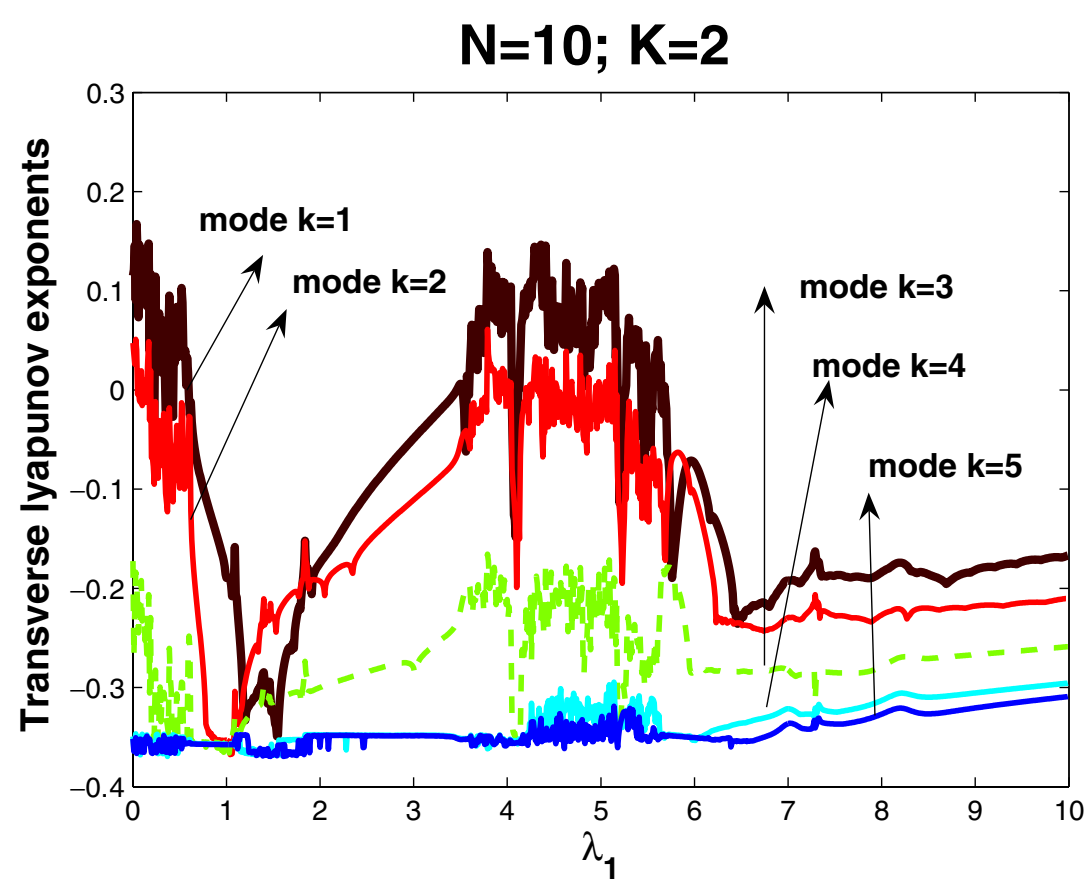

(b)

Fig. 8. Variations of the transverse Lyapunov exponents versus $\lambda_{1}$ with $w=1, \lambda_{2}=0.5$ and $\beta=\mu=5, E_{0}=3.5$.

\subsection{Results of the control}

\subsubsection{Results without parametric} perturbation $(q=0)$

The theory of the stability of the control of the unstable synchronization manifold is presented in the above subsection, the aim of the present subsection is to investigate the effects of the control on the synchronization manifold. This is to find the range of gain parameter $\lambda_{i}$ in which the control process is good. It is important to note that the choice of $K$ can been found in the unstable region for 
the uncontrolled ring of mutually coupled chaotic oscillators. One notices that, the role of the active control is to ensure that all arbitrary small transverse variations decay to zero, and consequently the controlled system develops a stable synchronization. On the other hand, we need to verify whether the active control has a positive or a negative effect on the unstable synchronization manifold, or find if the gain parameter introduces de-synchronization phenomena in the original system.

Figure 8 shows the variation of the Maximum transverse Lyapunov exponents as a function of the coupling parameter $\lambda_{1}$ for two values of the coupling coefficient $K$ which corresponds to the unstable synchronization, (i.e. $K=1$ and $K=2$ ). Table 2 presents the stability boundary of the control process in the plan $\left(\lambda_{1}, \lambda_{2}\right)$ for the ring of $N=10$ oscillators with the value of $\mathrm{K}$ choose in the unstable. As it appears, different types of dynamical states take place. In Fig. 8, we find that as $\lambda_{1}$ increases from zero with a fixed value of $\lambda_{2}$, (i.e. for example $\lambda_{2}=0.5$ ) the unstable synchronization exits until $\lambda_{1}=0.61$ where the bifurcation from unstable to stable synchronization appears, and remains until $\lambda_{1}=3.4$. Then after the value $\lambda_{1}=3.4$, the desynchronization transition appears and the system passes into unstable synchronization state, the control process of the unstable synchronization manifold is not achieved. As $\lambda_{1}$ increases further, the unstable synchronization state bifurcates to other stable synchronization states and the transition cascade continues leading to a stable and unstable synchronization state. In summary, for $\lambda_{2}=0.5$, it appears that the good gain coefficient $\lambda_{1}$, for controlling the unstable synchronization manifold is found in the region of $\lambda_{1}$ defined as $\lambda_{1} \in[0.6 ; 3.4] \cup[5.8 ; 14.1] \cup[15.1 ; 18.5]$. Table 2 shows that some regions of the gain coefficients lead to stable control of the unstable synchronization manifold.

\subsubsection{Effects of parametric perturbations on the control}

The previous subsection deals with finding the region of the gain parameters $\left(\lambda_{1}, \lambda_{2}\right)$ successfully and the results are shown in Table 2. To analyze the effects of the amplitude of parametric perturbations on the domain of the good gain parameters for the control process in the plane $\left(\lambda_{1}, \lambda_{2}\right)$, let us

Table 2. Some dynamical states in the mutually coupled chaotic systems under the control.

\begin{tabular}{lccc}
\hline$\lambda_{2}$ & Domains of $\lambda_{1}$ for Stable Control & $\lambda_{2}$ & Domains of $\lambda_{1}$ for Stable Control \\
\hline 0.1 & {$[2.8 ; 16.3] \cdots$} & 1.6 & {$[0.2 ; 1] \cup[1.7 ; 4.4] \cup[4.7 ; 6.7] \cdots$} \\
0.2 & {$[1.9 ; 8.5] \cup[14.2 ; 30] \cdots$} & 1.7 & {$[0.2 ; 1] \cup[1.7 ; 4.1] \cup[4.5 ; 6.1] \cdots$} \\
0.3 & {$[1 ; 5.6] \cup[9.6 ; 25] \cdots$} & 1.8 & {$[0.1 ; 0.9] \cup[1.6 ; 3.9] \cup[4.2 ; 5.8] \cdots$} \\
0.4 & {$[0.8 ; 4.2] \cup[7.2 ; 17.6] \cdots$} & 1.9 & {$[0.2 ; 0.8] \cup[1.6 ; 3.7] \cup[4 ; 5.5] \cdots$} \\
0.5 & {$[0.6 ; 3.4] \cup[5.8 ; 14.1] \cup[15.1 ; 18.5] \cdots$} & 2.0 & {$[0.2 ; 0.8] \cup[1.4 ; 3.5] \cup[3.8 ; 5.3] \cdots$} \\
0.6 & {$[0.6 ; 2,8] \cup[4.8 ; 11.8] \cdots$} & 2.1 & {$[0.1 ; 0.8] \cup[1.3 ; 3.3] \cup[3.6 ; 5.6] \cdots$} \\
0.7 & {$[0.5 ; 2.4] \cup[4.1 ; 10.1] \cup[10.7 ; 15] \cdots$} & 2.2 & {$[0.2 ; 0.8] \cup[1.3 ; 3.3] \cup[3.5 ; 4.8] \cdots$} \\
0.8 & {$[0.4 ; 2.1] \cup[3.6 ; 8.8] \cup[9.5 ; 13.4] \cdots$} & 2.3 & {$[0.2 ; 0.7] \cup[1.2 ; 3.0] \cup[3.3 ; 4.5] \cdots$} \\
0.9 & {$[0.1 ; 1.9] \cup[3.2 ; 7.9] \cup[8.4 ; 11.7] \cdots$} & 2.4 & {$[0.2 ; 0.7] \cup[1.2 ; 3.0] \cup[3.2 ; 4.4] \cdots$} \\
1.0 & {$[0.3 ; 1.7] \cup[2.9 ; 7.1] \cup[7.6 ; 10.5] \cdots$} & 2.5 & {$[0.2 ; 0.6] \cup[1.2 ; 4.2] \cdots$} \\
1.1 & {$[0.3 ; 1.5] \cup[2.6 ; 6.4] \cup[6.9 ; 8.5] \cdots$} & 2.6 & {$[0.2 ; 0.6] \cup[1.0 ; 4] \cdots$} \\
1.2 & {$[0.2 ; 1.5] \cup[2.6 ; 6.4] \cup[6.9 ; 8.5] \cdots$} & 2.7 & {$[0.2 ; 0.6] \cup[1.1 ; 3.9] \cdots$} \\
1.3 & {$[0.3 ; 1.3] \cup[2.3 ; 5.8] \cup[6.3 ; 8.8] \cdots$} & 2.8 & {$[0.2 ; 0.6] \cup[1.1 ; 3.7] \cdots$} \\
1.4 & {$[0.3 ; 1.2] \cup[2.1 ; 5] \cup[5.4 ; 7.5] \cdots$} & 2.9 & {$[0.2 ; 0.6] \cup[0.9 ; 3.6] \cdots$} \\
1.5 & {$[0.2 ; 1.1] \cup[2.0 ; 4.8] \cup[5.1 ; 7.0] \cdots$} & 3.0 & {$[0.1 ; 0.5] \cup[1.0 ; 3.5] \cdots$} \\
\hline
\end{tabular}

Table 3. Effects of the amplitude of the parametric perturbation on the domains of the stable control.

\begin{tabular}{cc}
\hline $\mathrm{q}$ & Domains of $\lambda_{1}$ for Stable Control $\left(\lambda_{2}=0.2\right)$ \\
\hline 0 & {$[1.5 ; 8.5] \cup[14.2 ; 30] \cdots$} \\
0.3 & {$[1.0 ; 5.3] \cup[6.0 ; 6.8] \cup[14.4 ; 29.3] \cdots$} \\
0.6 & {$[0.8 ; 3.6] \cup[4.1 ; 5] \cup[5.6 ; 5.9] \cup[8.7 ; 16.1] \cup[17.3 ; 17.5] \cup[21.1 ; 25.4] \cup[26.8 ; 27.3] \cdots$} \\
0.9 & {$[0.6 ; 2.7] \cup[3.1 ; 4.4] \cup[6.3 ; 11.5] \cup[16.2 ; 18] \cup[20.3 ; 21.7] \cup[22.7 ; 30] \cdots$} \\
\hline
\end{tabular}



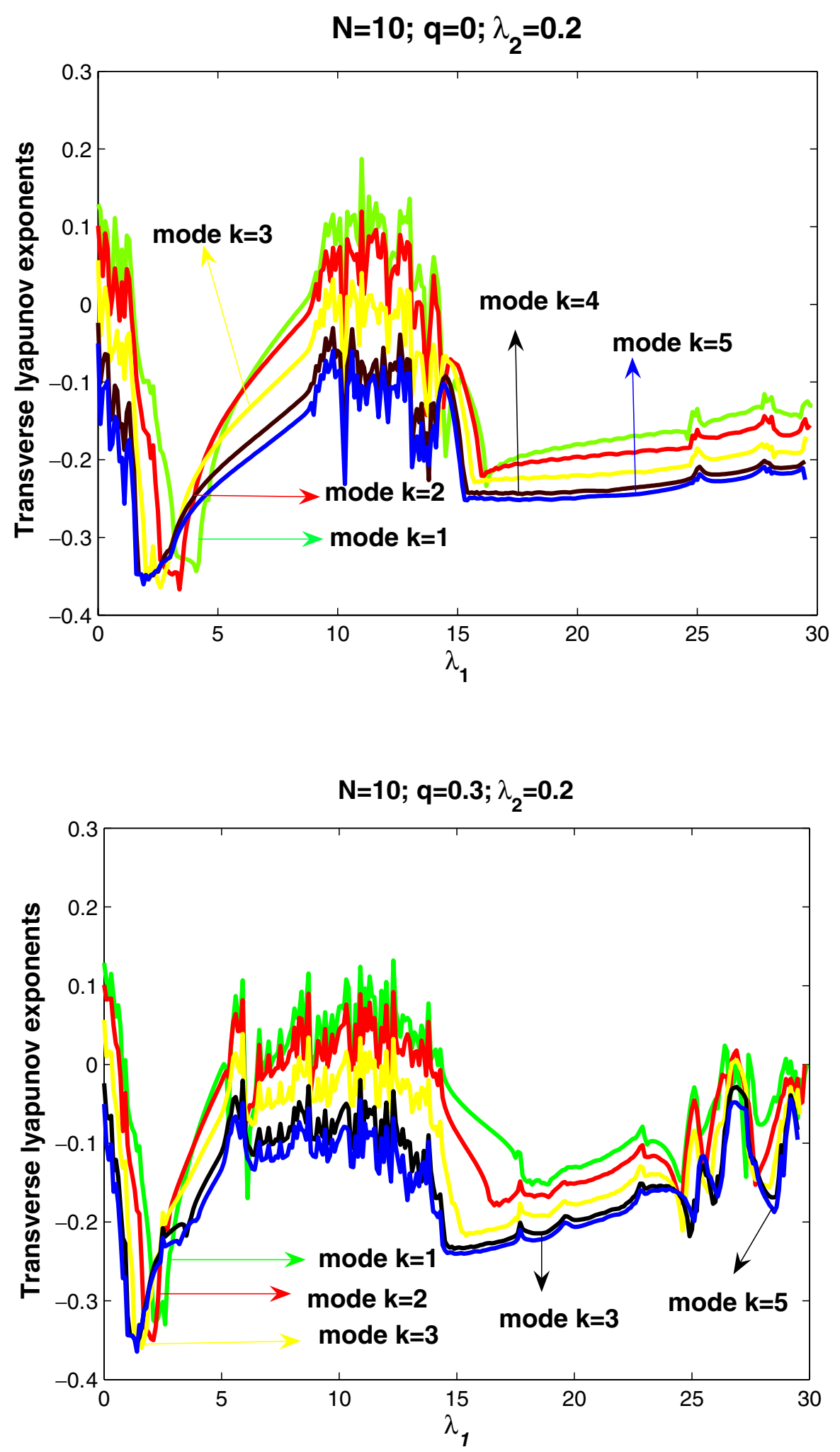

Fig. 9. Effects of the amplitude of the parametric coupling $q$ on the variations of the transverse Lyapunov exponents versus $\lambda_{1}$ with $w=1, \lambda_{2}=0.2$ and $\beta=\mu=5, E_{0}=3.5$. 

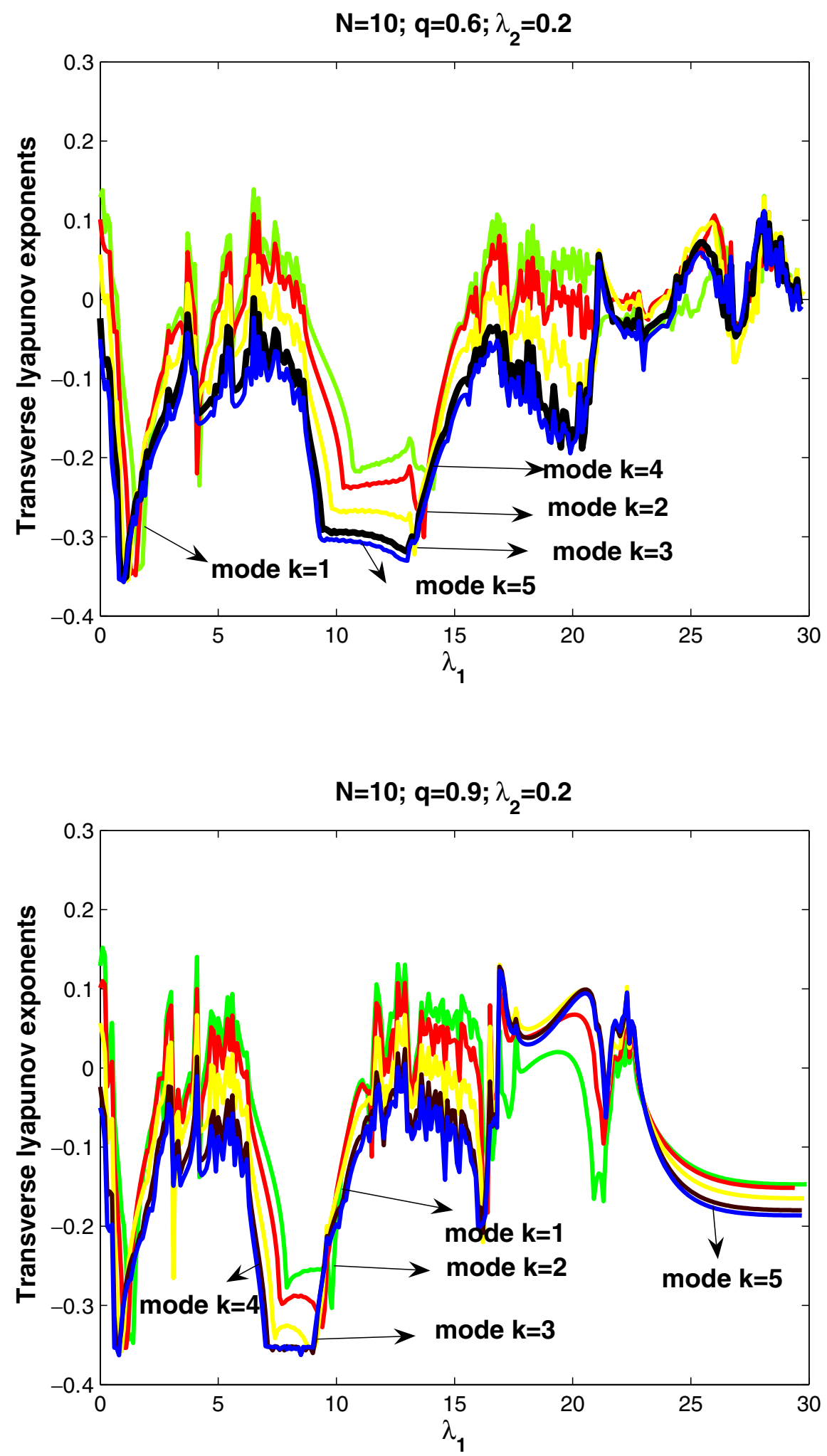

Fig. 9. (Continued) 


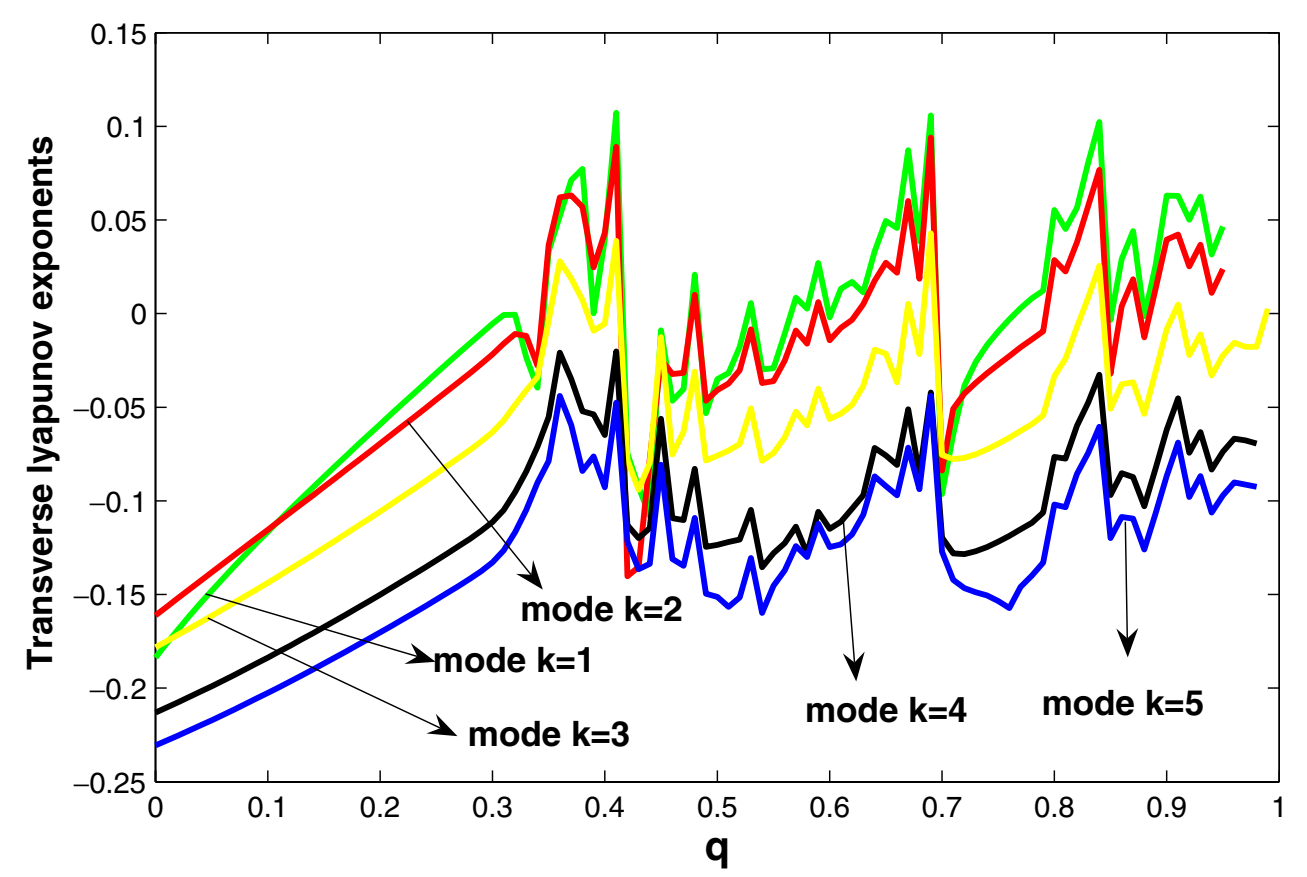

(a)

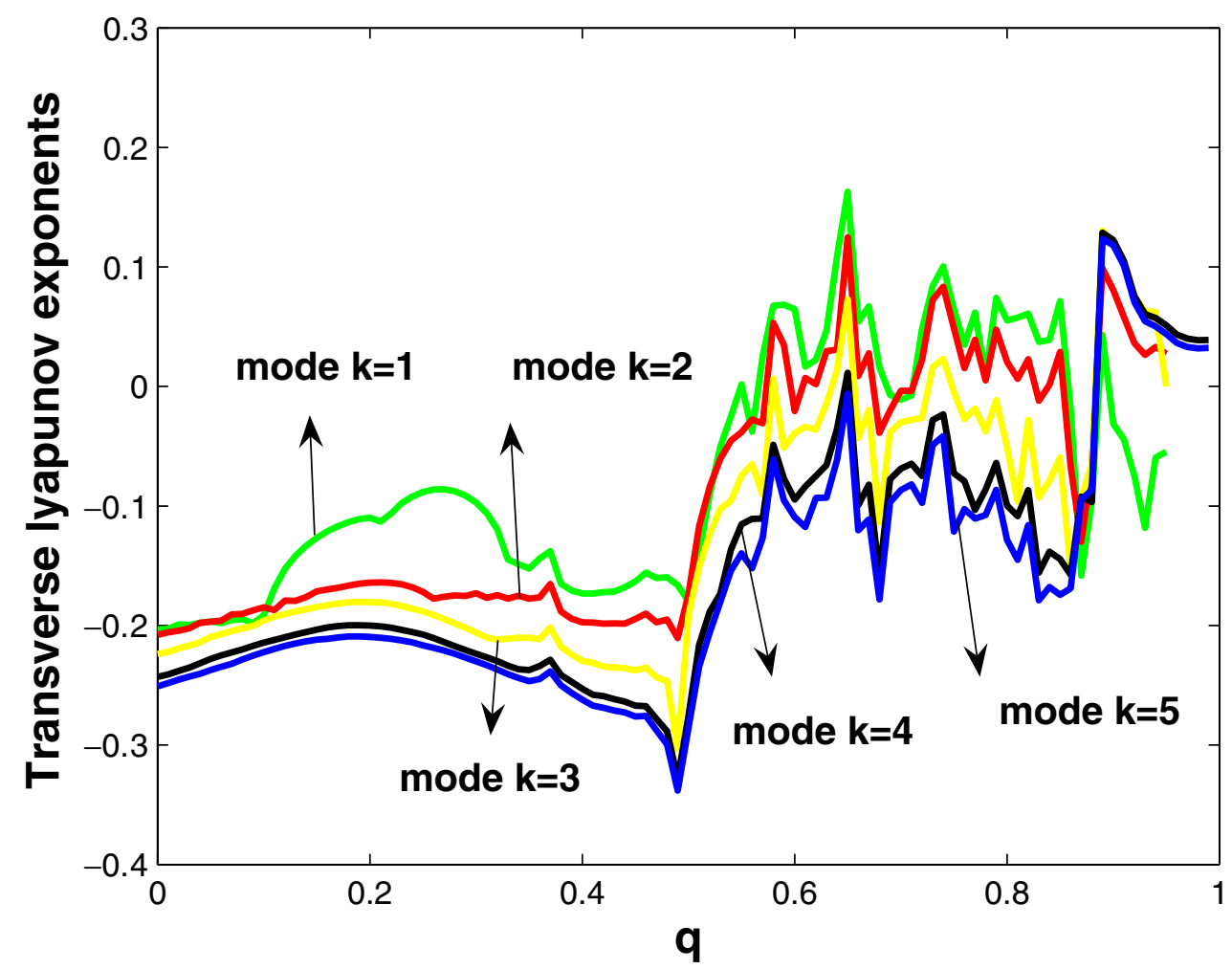

(b)

Fig. 10. Variations of the transverse Lyapunov exponents versus $q$ with $w=1$ and $\beta=\mu=5, E_{0}=3.5, \lambda_{1}=0.2, K=1$, (a) $\lambda_{1}=5$ and (b) $\lambda_{1}=17$. 
fix the value of one gain coefficient before carrying out our analysis. For example, for $\lambda_{2}=0.2$, the above investigation of master stability function approach enables to find that the control of the unstable synchronization manifold is stable for the value of $\lambda_{1}$ defined as $\lambda_{1} \in[1.5 ; 8.5] \cup[14.2 ; 20] \cdots$. Analyzing the effects of the parametric perturbations, we use three values of $q$ as $\lambda_{1}$ varies. When $q=0.3$, our investigations enable us to find that the control process of the unstable synchronization manifold in the ring is achieved for $\lambda_{1} \in[1.0 ; 5.3] \cup[6.0 ; 6.8] \cup[14.4 ; 29.3] \cdots$. For the other values of $q$, the results are shown in Table 3 , and we find that the domains of stable control of the unstable synchronization are strongly changed when the amplitude $q$ of the parametric perturbations increases. The situation also shows up in Fig. 9 where the variation of the transverse Lyapunov exponents are plotted versus the coupling gain parameter $\lambda_{1}$.

Figure 10 shows the variation of the transverse Lyapunov exponents versus $q$ with the values of the coupled gain parameters chosen in the region of stable control. We find here the negative effects of the parametric perturbations on the control.

\section{Conclusions}

We have studied in this Letter the possibility of controlling the unstable synchronization manifold on a ring of mutually coupled chaotic oscillators. Various bifurcation structures and transition for the chaotic states have first been found using numerical simulation. Stability boundaries for the synchronization process in a shift-invariant ring of oscillators have been derived following the master stability function approach, and the transverse Lyapunov exponents. Secondly, we have used the parametric active control strategy to stabilize the controlled system under variations of the gain parameters. The stability of the control process was done using the master stability function approach.

\section{References}

Boccaletti, S., Latorab, V., Moreno, Y., Chavez, M. \& Hwang, D. U. [2006] "Complex networks: Structure and dynamics," Phys. Rep. 424, 175-308.

Chen, M. \& Zhou, D. [2006] "Synchronization in uncertain complex networks," Chaos 16, 013101.

Filatrella, G., Straughn, B. \& Barbara, P. [2001] J. Appl. Phys. 90, 5675.
Fukui, K. \& Nogi, S. [1980] "Power combining ladder networks with many active devices," IEEE Trans. Microwave Th. Tech. MTT-28, 1059-1067.

Fukui, K. \& Nogi, S. [1986] "Mode analytical study of cylindrical cavity power combiners," IEEE Trans. Microwave Th. Tech. MTT-34, 943-951.

Hansel, D. \& Sompolinsky, H. [1992] "Synchronization and computation in a chaotic neural network," Phys. Rev. Lett. 68, 718-721.

Heagy, J. F., Carroll, T. L. \& Pecora, L. M. [1994] "Synchronous chaos in coupled oscillator systems," Phys. Rev. E 50, 1874.

Heagy, J. F., Carroll, T. L. \& Pecora, L. M. [1995] "Short wavelength bifurcations and size instabilities in coupled oscillator systems," Phys. Rev. Lett. 74, 4185-4188.

Jankowski, S., Londei, A., Mazur, C. \& Lozowski, A. [1995] Int. J. Electron. 79, 823.

Kuramoto, Y. [1984] Chemical Oscillations, Waves and Turbulence (Springer, Berlin).

Li, R. \& Erneux, T. [1993] Opt. Commun. 99, 196.

Li, R. \& Erneux, T. [1994] "Bifurcation to standing and traveling waves in large arrays of coupled lasers," Phys. Rev. A 49, 1301-1312.

Linkens, D. A., Taylor, I. \& Duthie, H. L. [1976] "Mathematical modeling of the colorectal myoelectrical activity in humans," IEEE Trans. Biomed. Eng. BME-23, 101-110.

Miyakawa, K. \& Yamada, K. [2001] "Synchronization and clustering in globally coupled salt-water oscillators," Physica D 151, 217-227.

Nakata, S., Miyata, T., Ojima, N. \& Yoshikawa, K. [1998] "Self-synchronization in coupled salt-water oscillators," Physica D 115, 313-320.

Nana Nbendjo, B. R. \& Yamapi, R. [2007] "Active control of extended Van der Pol equation," Commun. Nonlin. Sci. Numer. Simul. 12, 1550-1559.

Oksasoglou, A. \& Vavrim, D. [1994] IEEE Trans. Circuits Syst. 41, 6690.

Otsuka, K., Kawai, R., Hwong, S., Ko, J. \& Chern, J. [2000] "Synchronization of mutually coupled selfmixing modulated lasers," Phys. Rev. Lett. 84, 30493052.

Pasemann, F. [1999] "Synchronized chaos and other coherent states for two coupled neurons," Physica D 128, 236-249.

Pecora, L. M., Caroll, T. L., Johnson, G. A. \& Mar, D. J. [1997] "Fundamentals of synchronization in chaotic systems, concepts and applications," Chaos $\mathbf{7}$, 520-543.

Pecora, L. M. \& Carroll, T. L. [1998] "Master stability functions for synchronized coupled systems," Phys. Rev. Lett. 80, 2109-2112.

Reimann, P., Van den Broeck, C. \& Kawai, F. [1999] "Nonequilibrium noise in coupled phase oscillators," Phys. Rev. E 60, 6402-6406. 
Robertson-Dunn, B. \& Linkens, D. A. [1974] "A mathematical model of the slow-wave electrical activity of human small intestine," J. Med. Biol. Eng. 12, 750-757.

Strogatz, S. H. \& Stewat, I. [1993] Sci. Am. (Int. Ed) 68, 102.

Vannucchi, F. S. \& Boccaletti, S. [2004] Math. Biosci. Eng. 1, 49.

Winful, H. G. \& Rahman, L. [1990] "Synchronized chaos and spatiotemporal chaos in arrays of coupled lasers," Phys. Rev. Lett. 65, 1575-1578.
Yamapi, R., Chabi Orou, J. B. A. \& Woafo, P. [2003] "Harmonics dynamics and transitions to chaos in a non-linear electromechanical system," Physica Scripta 67, 269-275.

Yamapi, R. \& Bowong, S. [2006] "Dynamics and chaos control in the self-sustained electromechanical device with discontinuity," Commun. Nonlin. Sci. Num. Simul. 11, 355-375. 